\title{
Fractal Cracks Propagation in Aluminum
}

\author{
Francisco Casanova del Angel ${ }^{1}$, Jaime Retama Velasco ${ }^{2}$ \\ ${ }^{1}$ SEPI ESIA, Instituto Politécnico Nacional, Mexico City, Mexico \\ ${ }^{2}$ IINGEN, Universidad Nacional Autónoma de México Mexico City, Mexico \\ Email: fcasanova49@prodigy.net.mx,.JRetamaV@iingen.unam.mx
}

Received June 2, 2013; revised July 2, 2013; accepted July 10, 2013

Copyright (C) 2013 Francisco Casanova del Angel, Jaime Retama Velasco. This is an open access article distributed under the Creative Commons Attribution License, which permits unrestricted use, distribution, and reproduction in any medium, provided the original work is properly cited.

\begin{abstract}
The theory of the fractal structure characterizing propagation of a crack through identification of its generator is presented. It's generating fractal, the peculiarities of its construction and the way to measure its segments are defined, and a theorem on the inverse scale property of such and other of the axial symmetry property of the fractal generator are presented and demonstrated. The theory is applied on 6061-T6 aluminum samples, using SENB probes. Direction of crack propagation and its fractal dimension are calculated numerically. Results obtained from modeling the direction of crack propagation through mechanics of elastic linear fracture and the one proposed here, called geometrical fractal fracture, are compared, thus developing the mirror case.
\end{abstract}

Keywords: Geometrical Fractal Fracture; Crack Propagation; Aluminum; Stress Intensity Factor; Fractal Dimension

\section{Introduction}

Crack propagation in solids has been object of various studies to understand its behavior. In traditional fracture mechanics, failure surfaces are considered as smooth, which differs from observations. Quantitative description of fracture surface rugosity is a parameter leading to treat fractures as fractals, using a quantitative fractographic analysis [1] and [2]. There are works confirming that morphology of the crack surface implies a complex microstructures mosaic, with the following main features: fracture mode and microstructure of material. Through a fracture numerical model for a polymer, carried out by [3], it has been shown that for a symmetrical failure surface, fractal dimension has a universal $D=1.27 \pm 0.02$ value; then, [4] showed that for a kinetic theoretical model simulating propagation of the crack, the resulting fracture surface is a fractal, which dimension depends on the elastic constants of the material. In order to apply the theoretical development presented in the first part of this report, tests were carried out on 6061-T6 aluminum samples, with a combination of magnesium and silicon for thermal treatment. Such alloy has a high working and corrosion resistance, as well as low deformation. Most common alloys included in this group are: 6061, 6063, and 6082 , used in building construction and the transportation industry.

\section{Characterization of Dendrogramatic Fractal}

Definition of generating curve. Let $I_{0}$ be a unitary lenght line segment, contained in a closed interval, that is, $I_{0} \subset$ $[a, b]$. Let $I_{1}$ be a set with sectioned behavior, consisting in three segments of a straight line which create, based on starting point $a$ of $I_{0}$, two scalene triangles reflected regarding the middle point $c$ of $I_{0}$, obtained as follows: the first half of segment $I_{1}$ is substituted or removed by the sides of triangle which create an angle with $I_{0}$. This process is repeated for the second half, but with the sides reflected from middle point $c$.

This process is known as generator which is called state 1. Construction of set $I_{2}$ is made applying the generator to every segment of $I_{1}$, which is called state 2 . Thus, set $I_{k}$ is created applying generator $I_{1}$ on every segment of $I_{k-1}$, which is called state $k$, Figure 1(a).

Somer particular acpects presented in the construction of this type of fractal should be noticed when generator is created by combination or triangular layouts (in size and form):

- States $I_{k-1}$ and $I_{k}$ are different from each other in the sequence shown by poligonal curves $\forall k \rightarrow \infty$,

- set $I$ has a fine structure, that is, it contains every detail in every arbitrary small scale.

- although the generator is composed by two triangular figures corresponding to Euclidean logic, the geome- 
try of $I_{k} \forall k \rightarrow \infty$ is too irregular to be described in classical geometric terms.

Figure 1(b) shows the curve built on the plane through reflection of $I_{k}$ on every side.

Curve $I$ is characterized by being of a similar scale since, based on a transformation $F: \mathfrak{R}^{n} \rightarrow \mathfrak{R}^{n}$ with $\lambda_{i}>0 \forall i \quad \exists_{n} a, b \in \mathfrak{R}^{n}$ such that $\left|F_{\mathrm{i}}(a)-F_{\mathrm{i}}(b)\right|=\lambda_{i}|a-b|$. Similarity of scale is present

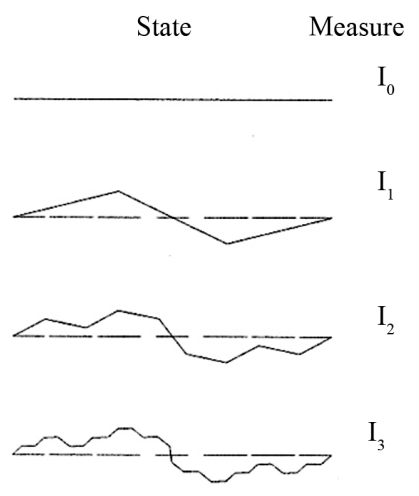

(a)

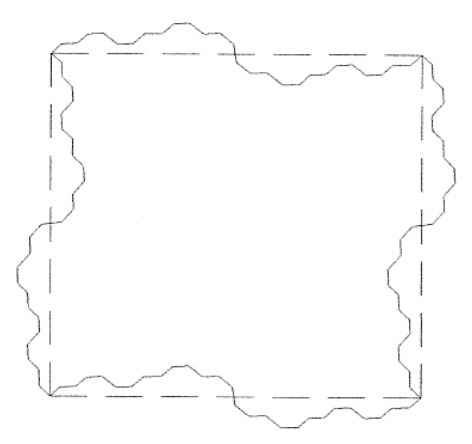

(b)

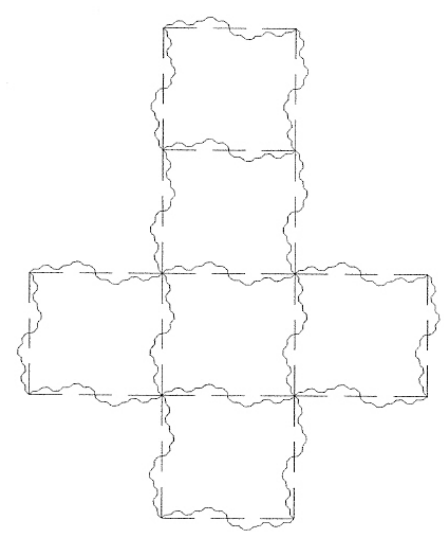

(c)

Figure 1. (a) Construction of the fractal curve $I$. In every state $I_{k}$ generator $I_{1}$ is applied on every segment of the curve. (b) Fractal curve $I$ on the plane, and (c) cubic form of factal. for triangles created with $I_{0}$, by generator $I_{1}$. In like manner, it also has the property to be affine since, based on transformation $F$ already defined, $F(a)=T(a)+\alpha$ with $T$ a non-singular linear transformation and $\alpha \in \mathfrak{R}^{n}$. It must be remembered that affinity is conceived as a shear transformation or resistant to cutting, and is a contracting or expanding effect, not necessarily in the same direction.

A curve type $l_{k}$ satisfies the scale principle if all its relative figures are linked to each other by a scale law.

Let $I$ be a Borel's set such that $I=\left\{I^{1}, I^{2}, I^{3}\right\}$ where $I^{\mathrm{j}}$ is a finite succesion of line segments creating the generator $\forall j=1,2,3$, in order that $I_{i}=\bigcup_{j=1}^{3} I_{i}^{j} \forall i=1,2, \cdots$

is a countable sequence of sets. Thus, measurement $\mu$, of segments $I_{\mathrm{i}}$ is defined as:

$$
\begin{aligned}
& \mu\left(\bigcup_{i=1}^{\infty} I_{i}\right)=\sum_{i=1}^{\infty} \mu\left(\bigcup_{j=1}^{3} I_{i}^{j}\right) \\
& =\mu\left(I_{i}\right)=3 \mu\left(I_{i-1}\right) \quad \forall i=1, \cdots
\end{aligned}
$$

When a geometrical discontinuity is of the fractal type, generated by a natural process, a uniform reticulate should be built. Let $(\chi, P(\chi), \mu)$ be a space with measures such that the sample space is $\chi=[0,1]$ and $P(\chi)$ is a set of subsets of $\chi$, where the measurement is $\mu$. Since the system is dynamic, $\chi \subseteq \mathfrak{R}^{p}$ is the phase space. Let us consider a $\chi$ reticulate covered by $p$-dimensional boxes with radius $\delta_{n}$, where $B_{\delta n}(t)$ is the neighbor box containing the segment of straight line or point $t$. Succession of neighbor boxes has radius $\delta_{n} \rightarrow 0$ as $n \rightarrow \infty$.

Let us suppose that there is subset $I \neq \phi$ from Euclidian space $n$-dimensional, $\mathfrak{R}^{n}$, and that

$|I|=\sup \{|a-b|$ such that $a, b \in I\}$. If $\left\{I_{i}\right\}$ is a countable set of neighbors with radius $\delta$ covering $I$, thus, there exists a subset $I I$ of $\mathfrak{R}^{\mathrm{n}}$ such that $I I \subset\left(\bigcup_{i=1}^{\infty} I_{i}\right)$ with $\left|I_{i}\right| \in[0, \delta] \forall i$. Therefore, $\left\{I_{i}\right\}$ is neighbor $\delta$ of $I$. If there is a $k>0$, then for every neighbor $\delta>0$ a function to minimize total covering $I I$ may be defined as follows:

$$
H_{\delta}^{k}(I I)=\inf \left\{\sum_{i=1}^{\infty}\left|I_{i}\right|^{k}\right\} \quad \forall\left\{I_{i}\right\} \text { neighborhood } \delta \text { of } I I
$$

If in (2) we consider the limit, then $\lim H_{\delta}^{k}(I I)=$ $H^{k}(I I)$. It must be taken into account that $H^{k}(I I)$ is known as Hausdorf's $k$-dimensional measurement.

Theorem (of inverted scale property). Let $F^{-1}$ be an inverted similar transformation of scale factor $k>0$, such that $F^{-1}: \mathfrak{R}^{n} \rightarrow \mathfrak{R}^{n}$. Si $\exists I I \subset \mathfrak{R}^{n}$ then $\lambda^{k} K^{k}\left(F^{-1}(I I)\right)=H^{k}(I I) \forall \lambda>0$ is complied with.

Dem: since $\left\{I_{i}\right\}$ is a countable set of neighbors $\delta$ covering $I$, if we apply the inverted similar transformation: $F^{-1}\left\{I_{i}\right\}=\left\{F^{-1}\left(I_{i}\right)\right\}$ which is a $\delta / \lambda=\lambda^{-1} \delta$ neighbor of $F^{-1}(I I)$, that is:

$\lambda^{k} H_{\delta / \lambda}^{k}\left(F^{-1}(I I)\right) \leq H^{k}(I I) \forall \lambda>0$. On the limit when $\delta$ 
$\rightarrow 0$, the above inequality becomes:

$$
H^{k}\left(F^{-1}(I I)\right)=\lambda^{-k} H^{k}(I I)
$$

The theorem herein proven allows us to reduce the length of a fractal object.

\subsection{Meshing and Definition of Fractal Outline}

Let $N\left(t^{*}, \Delta t^{*}\right)$ be the number of squares contained by reticulate, and $N(t, \Delta t)$ the number of squares intersected by the fractal curve. $D_{\theta^{\circ}}(I)$ shall be the fractal dimension, L the total length of the object, and 1 the length of every segment. Therefore, L/l quotient defines the number of subdivisions contained by every side of the intersected reticulate. These scale properties correspond to a fragmented fractal, and the multifractal [5, p. 45] concept is applied. Based on the above:

$$
N\left(t^{*}, \Delta t^{*}\right) \cong \Delta t^{*} \rho\left(t^{*}\right) t^{f\left(t^{*}\right)}
$$

where $t \in\left[t^{*}, t^{*}+\Delta t^{*}\right] . P\left(t^{*}\right)$ is the probability of distribution of intersection points $t \in\left[t^{*}, t^{*}+\Delta t^{*}\right]$ and $f\left(t^{*}\right)$ the fractal dimension of such points.

Considering a random generation of $f(t)$, the original curve is rotated to different angles, preferably constant, in order to calculate $D_{\theta}$ for every case.

$$
D_{\theta^{\circ}}=\frac{\sum_{i}\left\{D_{\theta^{\circ} i} \forall i=1, \cdots, n\right\}}{n}
$$

In order to rotate the original curve a certain number of times, let us consider mapping $M_{n}: \chi \rightarrow \mathfrak{R}$, where $M_{n}(t)=-\log \mu\left[B_{\delta n}(t)\right]$, if $m\left[B_{\delta n}(t)\right]>0$ then $C_{n}(t)$ is a re-scalated version of $M_{n}(t)$, that is:

$$
C_{n}(t)=\frac{M_{n}(t)}{-\log \left(\delta_{n}\right)}
$$

where $C_{n}$ describes the local behavior of $\mu$ measurement.

\subsection{Behavior Pattern}

The fractal behavior of a geometrical discontinuity takes us to the concept to of diagonal self-affinity diagonal. In order to define the pattern of the fractal generator, let us begin drawing straight lines from its base to the points where such curves all along its length, thus obtaining a fractioned curve. It is convenient to draw horizontal lines in case there is a change in its path behavior, in order to identify the affinity along such path.

Horizontal lines identifying the beginning of the generator, must show the feature of proportionality $d$ such that:

$$
d=\mathcal{L} \sin \beta \quad \text { where } \sin \beta=\frac{d}{\mathcal{L}} \text { and } \cos \beta=\frac{k}{\mathcal{L}}
$$

$\mathcal{L}$ is the length of the generator, $\mathcal{l}$ the length of every segment, and $\mathcal{N} / \mathcal{C}$ is the number of subdivisions contained by the generator. In order to observe the ideal behavior of the path, the scale relationship is defined as the average of the lengths of adjoining generators, that is, $\left(l_{1}+\ell_{2}\right) / 2$; Figure 2(a).

The scale factor of every rotation underwent by the generator or fractal curve, $s$, is defined as:

$$
s=\log _{10}(\mathcal{N}) \quad \forall \mathcal{N} \rightarrow \infty
$$

where the value of $\mathcal{N}$ is the average of the highest number of every rotation, that is:

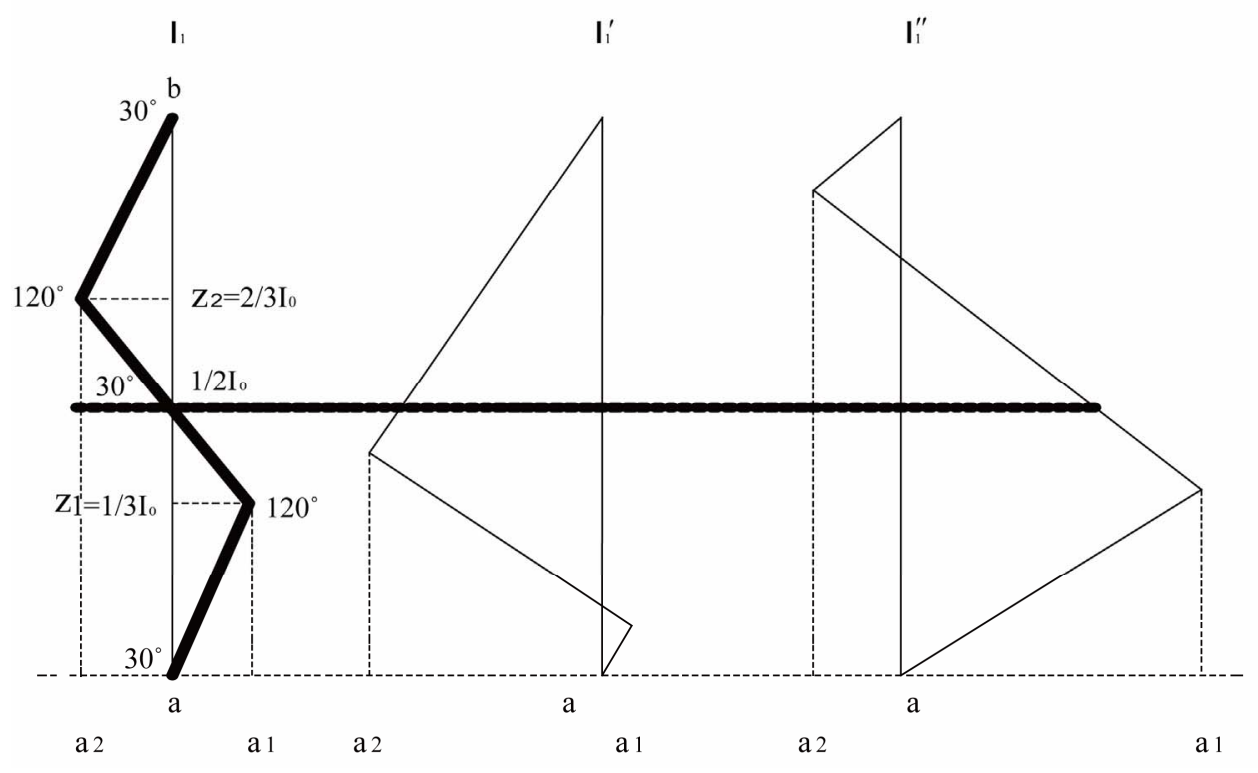

(a)

(b)

Figure 2. (a) Ideal generator; (b) Affinity of triangles in the generator. 


$$
\mathcal{N}=\sum_{i}\left\{R_{i} \quad \forall b \text { and } i=1, \cdots, n\right\}
$$

Based on the above, we may get the scale factor of the generator, defined as the inversion of $s$ which, applied, generates the geometrical structure.

\subsection{Parameters of Fractal Geometry}

Since the fact that the real length of a fractal line depends on accurateness of measurement is an important issue, the real total length of the line increases in accordance with equation number 8 :

$$
\mathcal{L}_{j+1}=\mathcal{L}_{j} * \mathcal{N} * n
$$

with $j$ as number of iterations, $N$ the number of elements in the base generator $(j=1)$ and $n$ the factor of reduction applied. Therefore, the real length of a fractal line $\left(\mathcal{L}_{j}\right)$ after $j$ iterations is given by equation number 9 :

$$
\mathcal{L}_{j}=\mathcal{L}_{p} * \varepsilon_{j}^{\left(d-D^{*}\right)}
$$

where $\varepsilon_{j}=n^{j}$ represents the lowest measurement unit in order to measure relative length of a segment of the fractal line. Parameter $d$ represents the Euclidian dimension of the object, $D^{*}$ is the fraction of the fractal dimension $D_{\theta^{\circ}}$ and $L_{p}$ is the length of the whole fractal, from one end to the other, without taking into consideration its roughness. In order to apply the above to fractals generated from a natural process $\varepsilon_{j}$ is cleared up from equation number 9 , thus obtaining:

$$
\varepsilon_{j}=\left(d-D^{*}\right) \sqrt{\frac{\mathcal{L}_{j}}{\mathcal{L}_{p}}}
$$

Property of axial symmetry of generator. Triangles creating segments of $I_{1}$, based on $I_{0}$ may vary regarding size and shape.

Generator $I_{1}$ may be considered as the union of two scalene Triangles $I I_{1}$ (triagle lower than $I_{0}$ ) and $I I_{2}$ (traingle higher than $I_{0}$ ) created based on $I_{0}$, and reflected on $1 / 2 I_{0}$, that is:

$$
\begin{aligned}
I_{1} & =\left\{(a, 0),\left(z_{1}, a_{1}\right),\left(z_{2}, 0\right),\left(z_{3}, a_{2}\right),(b, 0)\right\} \\
& =\left\{(a, 0),\left(z_{1}, a_{1}\right),\left(z_{2}, 0\right)\right\} \cup\left\{\left(z_{2}, 0\right),\left(z_{3}, a_{2}\right),(b, 0)\right\} \\
& =I I_{1} \cup I I_{2}
\end{aligned}
$$

When $z_{1}=1 / 3 I_{0} ; z_{2}=1 / 2 I_{0}$ and $z_{3}=2 / 3 I_{0}$ we talk about generator $I_{1}$, Figure 2(b) left. When $z_{1}<1 / 3 I_{0} ; z_{2}<1 / 2 I_{0}$ and $z_{3}<2 / 3 I_{0}$, we talk about $I_{1}^{\prime}$, Figure 2(b) right. When $z_{1}>1 / 3 I_{0} ; z_{2}>1 / 2 I_{0}$ and $z_{3}>2 / 3 I_{0}$, we talk about $I_{1}^{\prime \prime}$. In the two latter cases, the homologous angles creating the segments of the generator are congruent and their homologous sides are proportional, thus complying with the property of similarity of triangles, that is, $I_{1}{ }^{\prime}$ and $I_{1}{ }^{\prime \prime}$ are generators similar to $I_{0}$.
Theorem of transformation of linear affinity. If $G$ is a geometrical transformation of scale factors $r, s \in \mathfrak{R}$, such that $G: \mathfrak{R}^{n} \rightarrow \mathfrak{R}^{n}$, there are translation factors $h, k \in \mathfrak{R}$, which go back to $I_{m+1}$ an affine generator of $I_{\mathrm{m}}$.

Dem: let $r$ and $s$ be scale factors in $x$ and $y$ directions, respectively, of generator $I_{1}$, which may be described by Equation (1), that is, $I_{1}=3 \mu\left(I_{i-1}\right)$ with $i=1$. Since $r$ and $s$ are scale factors of $I_{1}$, then $I_{1} \times(r, s)$ increases $(\forall r, s>1)$, decreases $(\forall r, s<1)$ or is non-variable $(\forall r, s=1)$ and generator $I_{1}$ is similar. Generator $I_{1}$ may be re-scalated and translated if $\forall h, k \in \Re: I_{1}=I_{1} \times(r, s)+(h, k)$, therefore, $\forall m>1: I_{m}=I_{m} \times(r, s)+(h, k)=I_{m}$

By means of induction we have that, by Equation (1), for $m=1$ :

$$
\begin{aligned}
I_{1} & =I_{1} \times(r, s)+(h, k) \\
& =3 \mu\left(I_{0}\right) \times(r, s)+(h, k) \\
& =\mu\left(I_{1}\right) \times(r, s)+(h, k) \\
& =I_{1}
\end{aligned}
$$

If we consider the above is valid for $m>1$, then $\forall r, s$ $\neq 1, h$ and $k \neq 0$ :

$$
I_{m}=I_{m} \times(r, s)+(h, k)=I_{m}
$$

Let us demonstrate this for $m=m+1$.

$$
\begin{aligned}
I_{m+1} & =I_{m+1} \times(r, s)+(h, k) \\
& =3 \mu\left(I_{m}\right) \times(r, s)+(h, k) \\
& =\mu\left(I_{m+1}\right) \times(r, s)+(h, k) \\
& =I_{m+1}
\end{aligned}
$$

\section{Methods to Measure the Crack's Length}

An approximate measurement of the crack's length, $a$, is a requirement in order to obtain strength to fracture and to measure crack propagation due to stress. In addition to optical methods used to determine the point where the crack enters the surface, there are methods measuring the crack's length, generating a history of such measurements. Two of the most used methods to measure the crack's length are: potential drop and acoustic.

In this paper, cracks propagation in a solid, aluminum alloy 6061-T6, is been studied using the Fractal Geometry theory (FG). Firstly, toughness to fracture for failure Mode I was experimentally determined, and then, critical stress intensity factor was determined for Mode II using the results obtained by Henry Vaughan (1998). Then, direction of propagation was calculated in accordance with the LEFM theory (FM).Using the FG theory, fractal dimension of cracks has been determined for the studied aluminum, in order to relate it afterwards with facture properties of the material. Regarding propagation, a generator with which it is possible to determine the direction 
of crack propagation may be determined using a graphical procedure.

\subsection{Materials and Methods}

Experimental test have been carried out in order to measure fracture properties of the material, critical stress intensity factor for Mode I and propagation direction of the crack using the LEFM theory. Then, the same parameters are calculated using the FG theory in order to make a comparison analysis.

Materials. The tested material was structural aluminum alloy 6061-T6, which is a material thermally treated through magnesium and silicon. Chemical contents and mechanical properties are summarized in Tables $\mathbf{1}$ and $\mathbf{2}$, respectively. The key features of the aluminum alloys used are its high working and corrosion resistance, as well as low deformation.

Table 1. Chemical contents.

\begin{tabular}{cc}
\hline Component & $\mathbf{W}_{\mathbf{t}}(\mathbf{\%})$ \\
\hline Aluminum, $\mathrm{Al}$ & $95.8-98.3$ \\
Chromium, $\mathrm{Cr}$ & $0.04-0.35$ \\
Copper, $\mathrm{Cu}$ & $0.15-0.4$ \\
Iron, $\mathrm{Fe}$ & 0.7 maximum \\
Magnesium, $\mathrm{Mg}$ & $0.8-1.2$ \\
Manganese, $\mathrm{Mn}$ & 0.15 maximum \\
Silicon, $\mathrm{Si}$ & $0.4-0.8$ \\
Titanium, $\mathrm{Ti}$ & 0.15 maximum \\
Zinc, $\mathrm{Zn}$ & 0.25 maximum \\
Other, each & 0.05 maximum \\
Other, total & 0.15 maximum \\
\hline
\end{tabular}

Table 2. Mechanical properties.

\begin{tabular}{cc}
\hline Property & Value \\
\hline Density & $2.7 \mathrm{gr} / \mathrm{cm}^{3}$ \\
Tensile Strength, Ultimate & $310 \mathrm{Mpa}$ \\
Tensile Strength, Yield & $276 \mathrm{Mpa}$ \\
Modulus of Elasticity & $68.9 \mathrm{Gpa}$ \\
Poissons Ratio & 0.33 \\
Fatigue Strength & $96.5 \mathrm{Mpa}$ \\
Fracture Toughness & $29 \mathrm{Mpa}{ }^{*} \mathrm{~m}^{1 / 2}$ \\
Shear Modulus & $26 \mathrm{Mpa}$ \\
Shear Strength & $207 \mathrm{Mpa}$ \\
\hline
\end{tabular}

Experiments procedures. The type of geometry used is beam bending or three points (SENB). Its three characteristic dimensions are: length of the crack $a$, to the thickness $B$ and $W$ stilt. In Figure 3 we can see the geometry of the test specimen used as showing in Figure 4 is the machining end of the notch.

An approximate measuring of crack's length $a$, is required to obtain tenacity to fracture and to measure crack propagation due to fatigue. In addition to optical methods used to determine where the crack penetrates the surface, there are methods measuring crack's length generating a history for such measurements. Two of the most used methods to measure the crack's length are: potential fall and acoustical.

Material under study, Al 6061-T6, uses a magnesium and silicon combination for its thermal treatment. This alloy is highly working and corrosion resistant and shows low deformation. Most common alloys in this group are: 6061, 6063 and 6082, which are used in building edifices, and transportation industry. Let us see the definition of type of probe used, as well as its geometry, in order to comply with a flat deformation status. Its three characteristics dimensions are: crack's length $a$, thickness, $B$, and slope $W$.

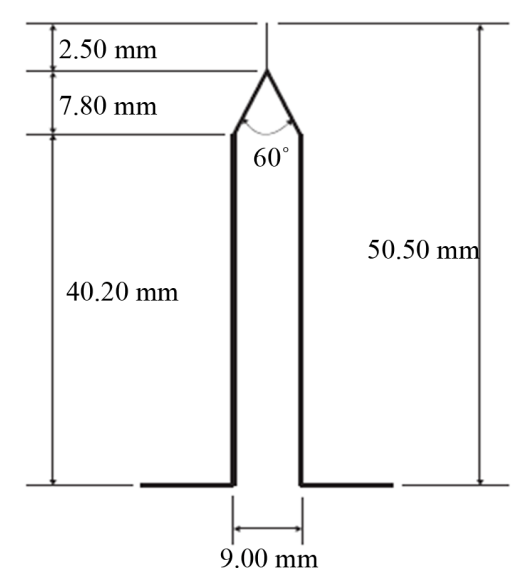

Figure 3. Geometry of the notch.

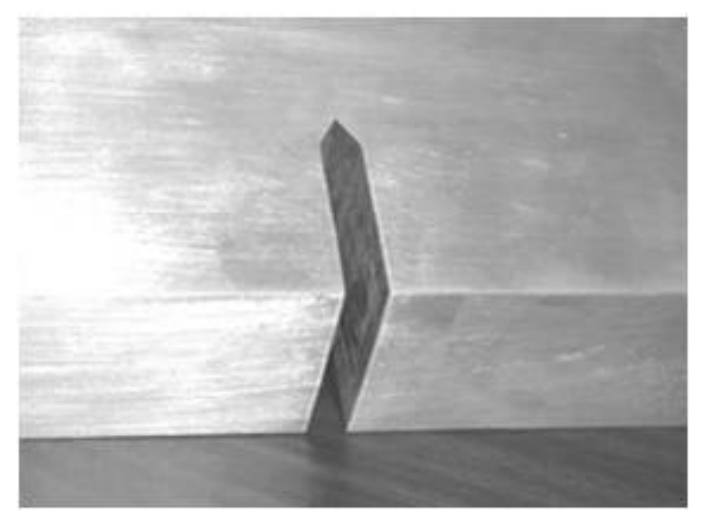

Figure 4. Specimen with the notch. 
In order that obtained values may be considered as valid, the following relationships must be complied with: $a, B \geq 2.5\left(K_{I C} / \sigma_{0}\right)^{2} ; 1 \leq W / B \leq 4 ; S=4 W ; L=4.2 W$, where the crack's length a is $50.5 \mathrm{~mm}$, thickness $B$ is 50 $\mathrm{mm}$, width $W$ is $100 \mathrm{~mm}$, and spacing between support $S$ is $400 \mathrm{~mm}$.

Referred Standard establishes that total length of the crack has a length induced by means of means of a special machining and another one induced by fatigue. This guarantees an elastic-linear behavior of the material. Machined crack is Chevron type, with a total length of $a$ $-0.025 \mathrm{~W}$. Regarding the crack by fatigue, such was induced applying a dynamic sinusoidal load, as specified by the Standard. The crack's length due to fatigue is $0.025 \mathrm{~W}$. Figure 5 shows the sinusoidal dynamic load used, and Figure 6 show the experimental results.

\subsection{Test Results}

The fracture toughness test was conducted in accordance with [6] and [7]. The environmental temperature in the moment was $25^{\circ} \mathrm{C}$ and humidity was $50 \%$.

Fracture toughness. The calculation of critical stress intensity factor of the material for Mode I, which establishes some limits regarding dimensions of probe, in order to avoid the generation of a plastic area on the tip of the crack, which dimensions influence the global behave-

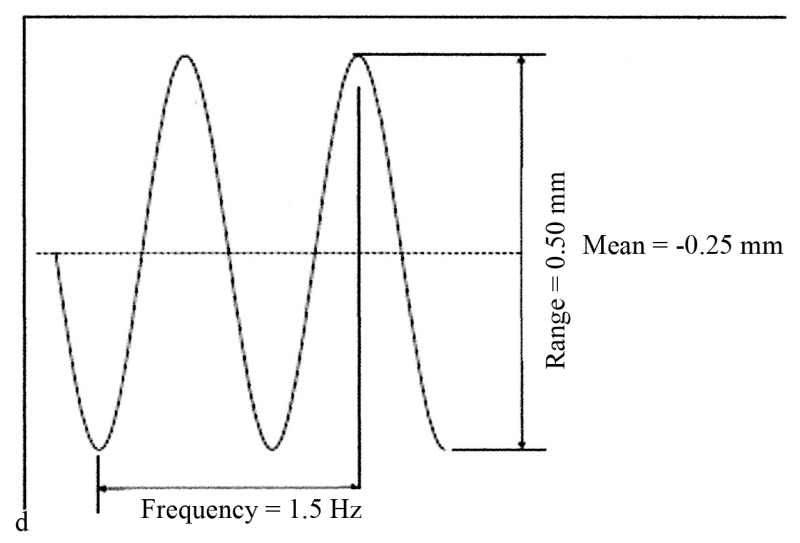

Figure 5. Sinusoidal load.

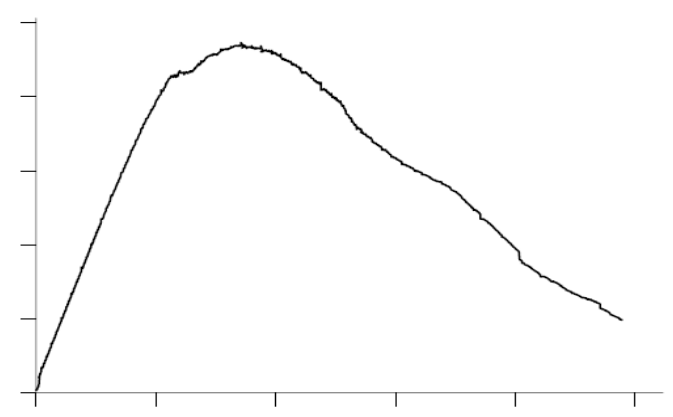

Figure 6. Experimental results. Load vs displacement. ior of the solid. Calculation of the toughness is carried out based on the load curve against displacements experimentally determined. Refer to [8] for a detailed description of calculation of such factor. After applying the theory established by the above Standard, stress intensity factor for aluminum alloy 6061-T6 is determined as: $K_{I C}=29.47 M P_{a} \sqrt{m}$.

Relationship between $K_{I C}$ and $K_{I I C}$. The effect of a mixed load mode for fractures has been particularly studied, since it is difficult that a material fails only due to a single mode in an independent manner in the practice. In numerical modeling of fracture, it is considered that the material fails first in Mode I and evolves to the remaining modes. The relationship between Modes I and II, which was experimentally studied by [9] is shown below.

For a material failing in accordance with the criterion of the main stress and load condition is only in Mode I, the failure occurs when:

$$
\left(\sigma_{0}\right)_{\max }=\frac{K_{I C}}{\sqrt{r}}
$$

In case the load condition generates a displacement of failure surfaces, Mode II, the failure occurs if:

$$
\left(\sigma_{0}\right)_{\max }=\frac{2 K_{I I C}}{\sqrt{3 r}}
$$

Equaling Equations (11) and (12), a relationship between the stress intensity factor for Modes I and II of failure is obtained; such being established as:

$$
K_{I I C}=\frac{\sqrt{3}}{2} K_{I C}
$$

Substituting values in Equation (13), critical stress intensity value is obtained for failure Mode II with the following value: $K_{I I C}=25.52 M P_{a} \sqrt{m}$

\subsection{Propagation of the Crack According to the LEFM Theory}

There are three numerical methods to calculate propagation direction of the crack [10]. The maximum main stress criterion has been used. Such criterion establishes that the crack propagates in a direction perpendicular to the direction of principal Stress I. Propagation begins when maximum main Stress I reaches the maximum tension stress of the material. Propagation direction of the crack is obtained with Equation (14).

$$
\theta=\tan ^{-1}\left[\frac{K_{1} \pm \sqrt{K_{1}^{2}+8 K_{11}^{2}}}{4 K_{1}}\right]=42.35
$$

Propagation direction of facture experimentally obtained is $36^{\circ}$ which, compared to that obtained using 
Equation (14), shows a $6.35^{\circ}$ variation. Figure 7 shows the propagation direction of fractures obtained in the laboratory.

\subsection{Computation of the Fractal Dimension}

In order to calculate the fractal dimension of crack generated, the box counting method applied in a graphical manner was used. The original crack was rotated at various degrees in order to determine in a reliable manner the fractal dimension $D$, since such must be the same in every case. The process carried out in order to determine $D$ was to choose a picture, Figure 8, which outline might be vectorized. Once the outline was draw, it was delimited in a rectangle, the required meshing for calculation of fractal dimension were built, calculation s were carried out and the results were graphed.

In order to obtain the fractal dimension of every graphed curve, a trend line was calculated, extrapolated backwards and its equation was calculated, obtaining its corresponding slope [11] and [12]. This is the fractal dimension of the fracture and Figure 9 shows such graphics. In order to obtain final dimension $D$, an average of dimensions of every of the four rotations applied

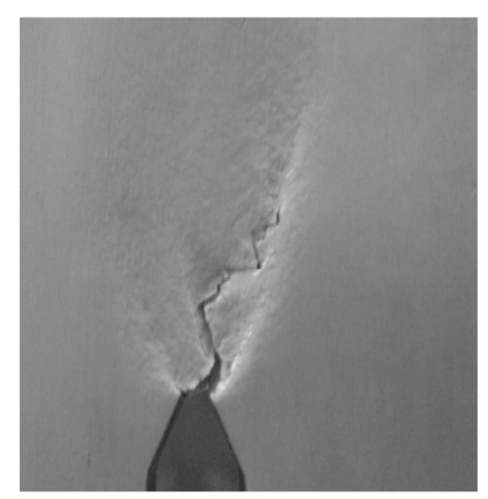

Figure 7. Fractal behavior of the crack.

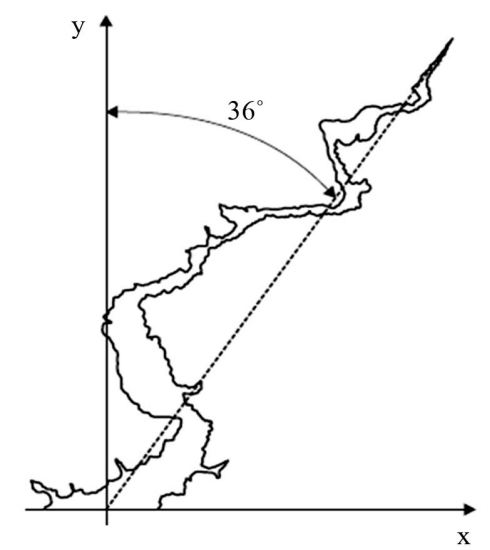

Figure 8. Propagation direction of factures obtained in the laboratory. on the fractal curve was calculated, concluding that fractal dimension for a crack in Al-6061-T6 is:

$D=\frac{\left(D_{0^{\circ}}+D_{45^{\circ}}+D_{90^{\circ}}+D_{135^{\circ}}\right)}{4}=1.2435$

Therefore, fractal dimension of cracks or fractures in Al 6061-T6 is $1.2435 \pm 0.0085$. This value of fractal dimension is used to relate FM to FG in determining the critical stress intensity factor for Modes I and II from FG theory, using the fracture dimension.

Figure 10 shows that the crack in the aluminum has the auto-affinity property characterizing fractal bodies generated by means of a natural propagation process, and there is a pattern repeating itself throughout it. From the basis middle point, straight lines have been drawn to the middle points of every fractioned curve along its path, thus obtaining a fractioned curve denoted by means of a thick gray line. When identifying a change on its path, horizontal lines have been drawn. This sectioned behaveior has been manifested up to four times all along its path, Figure 11(a), shown affinity along its path. The best delineated line has been defined as curve generator and its ideal form is shown in Figure 11(b).

\subsection{Direction of Fractal Propagation of the Crack}

Propagation direction of the crack was numerically determined by means of FM theory, considering that such is propagated perpendicular to maximum main stress I. Line of trend of propagation direction of the crack was carried out based on roughness compensation philosophy on both sides of such.

Fractal propagation direction of the crack has been determined under the following two considerations:

- Case in which a mirror effect is applied on even iterations of the generator, thus determining a $\theta=39^{\circ}$ propagation angle regarding the vertical, Figure 12(a); and

- Case where the mirror effect is not applied on even iterations, thus obtaining a $\theta=27^{\circ}$ propagation angle regarding the vertical, Figure 12(b).

With the first conditions a better modeling of crack's behavior is obtained, since experimentally a $\theta=36^{\circ}$ was obtained.

Table 3 shows results obtained by modeling propagation direction of the crack by means of FM and FG. In like manner, it is observed that, when modeling propagation direction of the crack by means of mirror FG case, a good approach to real behavior is obtained, as well as its corresponding modeling by means of FM. In accordance with crack's modeling, it is possible to predict its behavior, as well as its possible propagation direction, using an iterative auto-similarity process, feature of fractals, in accordance with the generation pattern in Figure 12(a) 


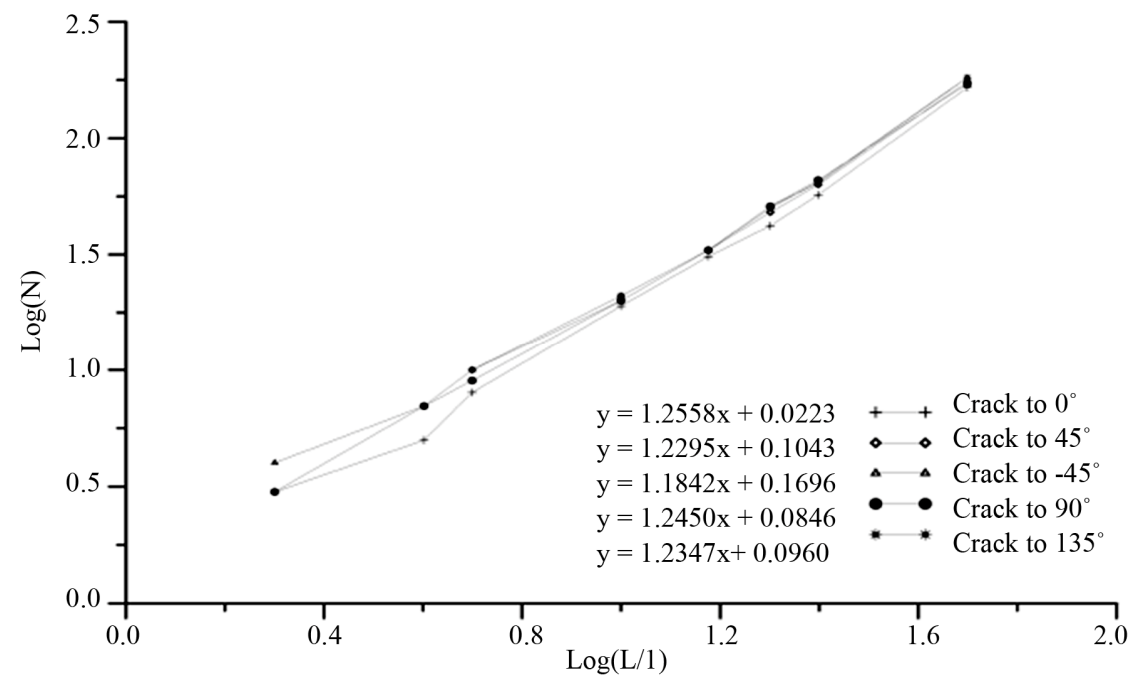

Figure 9. Graphic for calculation of fractal dimension.

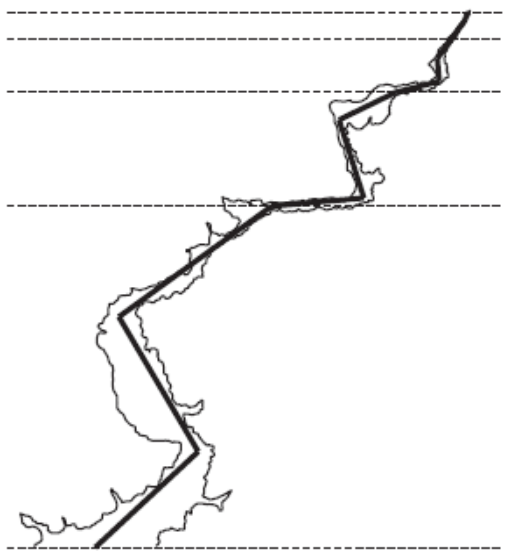

Figure 10. Crack in the aluminum alloy 6061-T6 seen as a fractal.

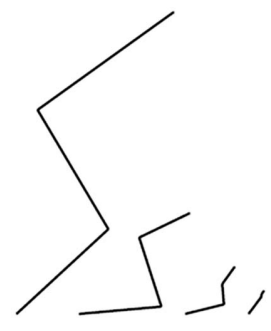

(a)

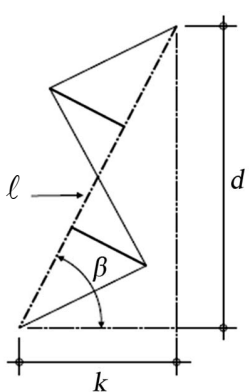

(b)
Figure 11. (a) Sectioned behavior of the crack and (b) ideal generator.

and the scale factor already obtained. Geometry obtained from such calculations was translated to the tip of the crack experimentally obtained, thus establishing a possible behavior of such facing possible loads which might continue to propagate it.

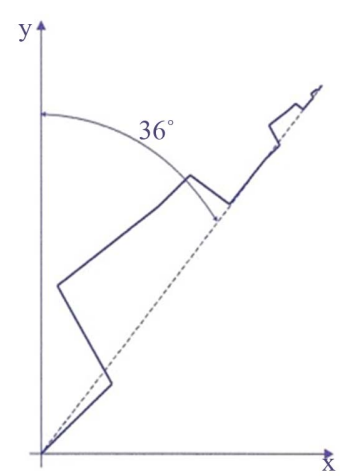

(a)

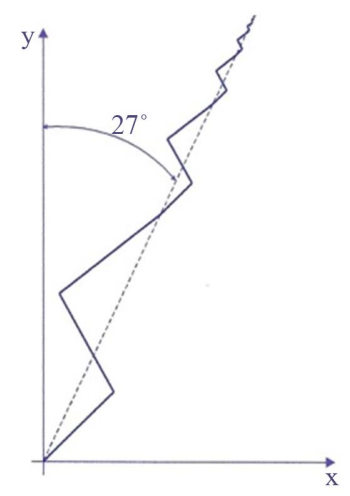

(b)
Figure 12. Fractal propagation direction of the crack. a) Mirror odd generator and b) Generator without rotation.

Table 3. Propagation direction.

\begin{tabular}{cccc}
\hline Modeling & $\theta^{\circ}$ & Variation $\left(^{\circ}\right)$ & Variation (\%) \\
\hline Experimental & 36 & & \\
LEFM & 42.35 & 6.35 & 17.63 \\
Mirror FG & 39 & 3 & 8.33 \\
FG without mirror & 27 & 9 & 33.33 \\
\hline
\end{tabular}

Figure 13 shows the crack obtained in the laboratory and its corresponding modeling by means of fractal theory. The gray line represents propagation of the crack experimentally obtained, which has been modeled by means of fractal theory; the black line above the horizontal, represents fractal behavior expected, which defines a $39.10^{\circ}$ future propagation angle regarding the vertical, from the tip of experimental crack.

Fractal geometry parameters. Since the fractal body under studied was generated from a natural crack propa- 
gation process in a solid body, the Equation (9) is applied.

Fractal dimension of the object under study previously obtained is $D=1.2435$. Therefore, $D^{*}=0.2435$. From Figure 14(c), lengths $L_{j}$ and $L_{p}$ are obtained, which values are: 113.0989 and 87.0268, respectively (the Figures 14(a) and (b) are the geometry of the crack behavior scaled and with mirror). These two values are taken in length values, regardless of the unit system used, and are determined in the following manner. $L_{j}$ is measured taking into consideration ins and outs of the line and $L_{p}$ is the end-to-end distance of referred figure. Therefore:

$\varepsilon_{j}=\sqrt[(1-0.2435)]{\frac{113.0989}{87.0268}}=1.41396$.

\section{Discussion}

Results obtained for critical stress intensity factor in Mode I, differ comparatively slightly from those reported by other works. This was achieved adhering to the standard. As for precision of probe's dimensions, it was observed that proper measurement of such, particularly regarding the length of the crack, is very important, since

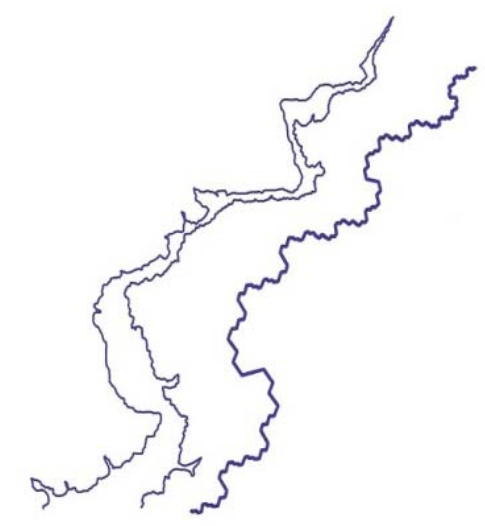

Figure 13. Modeling of the crack propagation by means of FG theory.

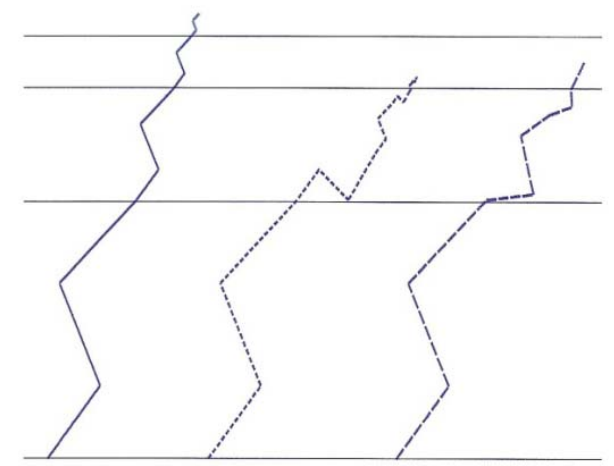

(a)

(b)

(c)

Figure 14. Geometry of the crack behavior. (a) Scaled, (b) With mirror, and (c) Experimental. cutting of material should be carried out in a laboratory guaranteeing an almost perfect cut or, preferably, in a numerical controlled lathe.

To create the crack due to stress, the importance of the machined crack's end finishing was observed since, if the curvature radius tends towards zero, the stress crack is induced faster. Numerical determination of fractal dimension of the crack in 6061-T6 aluminum alloy considered as a solid body results in a \pm 0.011 exactness through the box method.

Outline's rugosity orientation does not affect fractal dimension for 6061-T6 aluminum alloy, that is, fractal dimension for any rotation angle of the mesh is considerably the same for every case. Emphasizing properties characterizing fractal bodies (self affinity or self similarity, full-scale details, fractional dimension, among others), it may be considered that crack on 6061-T6 aluminum alloy shows behavior similar to that of those geometrical bodies. Therefore, it may be considered and treated with fractal geometry for its analysis and behaveior.

Fracture properties of $K_{I C}$ and $K_{I I C}$ materials and fractal dimension of the crack may be related, since topology of the fracture line (rugosity) directly influences fracture parameters of the material. Study of the fracture problem in metallic materials, 6061-T6 aluminum alloy, may be satisfactorily studied using set modeling through elastic linear fracture mechanics and fractal geometry. One of the advantages to model the fracture problem through fractal geometry is that it is capable to relate the macroscopic scale with the microscopic scale considering rugosity of the fracture line. It is known, based on works by other researchers, that rugosity of fracture line is directly related to atomic properties of the material. Therefore, fractal dimension for cracks on 6061-T6 aluminum alloy is a characteristic parameter.

An important issue observed when cyclic load is applied, is to know the quality of material used, since such may have imperfections directly affecting propagation of the crack. The origin of the material of the probe should be known; whether it is a cold laminated profile or cast with final geometry. It is recommended that probe comes from a laminated profile in order to guarantee homogeneity and isotropy of material.

It is recommended to decrease the geometry of probes to be used, in order to have a family of specimens to carry out a statistical analysis of data in order to determine desired properties. Based on laboratory observations, it is recommended that the curvature radius on the end of the machined crack tends to zero and frequency to apply cyclic load is $2 \mathrm{~Hz}$. An alternative to relate fractal dimension with fracture parameters of material, is to carry out a statistical analysis of data with a minimum of six probes, in order to carry out linear regression to find 
attempted ratio.

\section{Conclusions}

LEFM application leads to a determining analysis of propagation direction of the crack without taking into consideration intrinsic morphology in the cracking process of a solid; however, experimental analyses show that propagation direction does not depend only on facture properties of the material, molecular constitution of the material also contributes, since possible manufacturing defects, micro fissures and/or cavities lead to its coalescence to generate macro cracks through which energy shall be dissipated. It is possible to take into account roughness of the crack's walls by means of a fracture analysis using FG in order to calculate their nearest or experimentally observed propagation direction.

The property of axial symmetry of the generating curve proves the affinity of triangles created by such, with state zero. Characterizing the generating curve as a countable sequence of sets, allows us to prove the existence of the transformation to linear affinity of such. The generator must not be composed exclusively or necessarily by three segments, but it must be composed, at least, by two segments. In the cases presented, the generator or generating curve is composed by three line segments.

From the mechanical point of view, the error obtained when determining in the laboratory the critical stress intensity factor for Mode I was $1.62 \%$ regarding those reported in the literature. Obtaining the propagation direction applying LEFM shows a $17.63 \%$ variation, related to such obtained in the laboratory. Fractal dimension for aluminum 6061-T6 alloy is $1.2435 \pm 0.0085$. The crack in such structural aluminum shows a diagonal self-affine fractal behavior, with a 0.44668 scale factor and the direction of fractal crack propagation, mirror case, has a $8.33 \%$ variation, related to the experimental one.

\section{Acknowledgements}

This document was developed with part of the time devoted to the IPN-SIP 20120585 research project.

\section{REFERENCES}

[1] C. Guerrero and V. González, "Fractales: Fundamentos y Aplicaciones, Parte I: Concepción Geométrica en la
Ciencia e Ingeniería," Ingenierías,Vol. 4, No. 10, 2001, pp. 53-59.

[2] C. Guerrero and V. González, "Fractales: Fundamentos y Aplicaciones, Parte II: Aplicaciones en Ingeniería de Materiales," Ingenierías, Vol. 4, No. 12, 2001, pp. 15-20. www.uanl.mx/publicaciones/ ingenierias/12/

[3] Y. Termonia and P. Meakin, "Formation of Fractal Cracks in a Kinetic Fracture Model," Nature, Vol. 320, No. 6061, 1986, pp. 429-431. doi:10.1038/320429a0

[4] G. Peng and D. Tian, "Fracton Dimensionalities for Field-Biased Diffusion-Limited Aggregation Clusters," Physics Review B, Vol. 43, No. 10, 1991, pp. 8572-8575. doi:10.1103/PhysRevB.43.8572

[5] B. B. Mandelbrot, "Fractales, Hasard et Finance," Flammarion, Paris, 1997. doi:10.1007/978-1-4757-2763-0

[6] J. Oliver, "Modeling Strong Discontinuities in Solid Mechanics via Strain Softening Constitutive Equations. Part 1: Fundamentals," International Journal for Numerical Method in Engineering, Vol. 39, No. 21, 1996, pp. 35753600 .

doi:10.1002/(SICI)1097-0207(19961115)39:21<3575::AI D-NME65>3.0.CO;2-E

[7] J. Oliver, "Modeling Strong Discontinuities in Solid Mechanics via Strain Softening Constitutive Equations. Part 2: Numerical Simulation," International Journal for Numerical Method in Engineering, Vol. 39, No. 21, 1996, pp. 3601-3623.

doi:10.1002/(SICI)1097-0207(19961115)39:21<3601::AI D-NME64>3.0.CO;2-4

[8] J. Retama Velasco, "Mecánica de Fractura Fractal en Aluminio Structural," Master Thesis, Instituto Politécnico Nacional, México, 2006.

[9] H. Vaughan, "Crack Propagation and the Principal Tensile-Stress Criterion for Mixed-Mode Loading," Engineering Fracture Mechanic, Vol. 59, No. 3, 1998. pp. 393-397. doi:10.1016/S0013-7944(97)00138-0

[10] G. Juárez Luna, “Aplicación de la Mecánica de Fractura a Problemas de la Geotecnia," Master Thesis, Instituto Politécnico Nacional, México, 2002.

[11] V. Talanquer, "Fractus, Fracta, Fractal: Fractales, de Laberintos y Espejos," No. 47, Fondo de Cultura Económica, México, 2002.

[12] H. O. Peitgen, H. Jürgen and D. Saupe, "Fractals for the Classroom, Introduction to Fractals and Chaos, Part two," 2nd edition, Springer-Verlag, Berlin, 1993. 